\title{
Application of the Transition Probability Matrix Method to High Knudsen Number Flow Past a Micro-Plate
}

\author{
Andrew J. Christlieb*, W. Nicholas G. Hitchon ${ }^{\dagger}$, Quanhua Sun* and Iain D. Boyd* \\ ${ }^{*}$ Department of Aerospace Engineering \\ University of Michigan \\ 1320 Beal Avenue \\ Ann Arbor, MI 48109-2140 \\ ${ }^{\dagger}$ Department of Electrical and Computer Engineering \\ University of Wisconsin \\ 1415 Engineering Dr. \\ Madison, WI 53706
}

\begin{abstract}
In this work we present numerical results for the problem of 'high' (of order unity) Knudsen number gas flow past a micro-airfoil, for low flow velocity. The results are generated using an enhanced version of the transition probability matrix (TPM) method. The TPM is a non-statistical kinetic method [1] for computing neutral particle transport in high Knudsen number flows. The problem of high Knudsen number, low Mach number gas flow has been studied in the past using several computational approaches, such as the Information Preservation (IP) method [2] and the direct simulation Monte Carlo (DSMC) method [2]. For low Mach numbers, the DSMC approach suffers from statistical noise [3]. The IP method extends the range of the particle method by reducing the statistical noise of the approach. The need for a method which is capable of describing the particle distribution function for high Knudsen number flows at low flow velocities has led to an investigation of alternative kinetic approaches, such as the IP[4]. In this paper we present an altogether different approach to the problem of statistical noise, the transition probability matrix (TPM) method $[1,5,6,7]$. We give a brief overview of the TPM method, and compare its strengths and weaknesses to those of the IP and DSMC methods. Finally, we present results for the micro-plate and compare them to the results generated by both the IP and DSMC methods.
\end{abstract}

\section{INTRODUCTION}

In this work we consider a low velocity flow (Mach number, $M$, less than 0.3 ) past a flat plate of length of the order of $\frac{1}{10} \mu \mathrm{m}$. Such flows present challenges for well established statistical approaches, such as the direct simulation Monte Carlo (DSMC). At low Mach numbers, particle based approaches require a large number of samples in order to reduce statistical scatter to a level where the flow can be resolved (sample size $\sim 10^{5}$ for a variance less than $1 \frac{\mathrm{m}}{\mathrm{sec}}$ ). The need for a large number of samples can render these methods impractical [3]. Figure 1 illustrates the issue for a DSMC simulation. The flow is for a Mach number of $M=0.12$ and a Knudsen number of $K_{n}=0.05$, where $K_{n}=\frac{\lambda}{\text { PlateLength }}$. Adaptations of statistical methods have extended their range to cover these types of flows. One such adaptation is the information preservation (IP) method $[8,2,3,4]$. The IP method is a hybrid model that combines a particle (microscopic) description with a global (macroscopic) description. Although this extension works well for flows where $K_{n}<1.0$, the continuum model used in adjusting the global information becomes of questionable validity for $K_{n} \geq 1.0$.

Another approach to this problem of statistical noise is to develop a non-statistical (no random numbers used) kinetic model suited to these types of flows. To that end, we have developed a particle transport model based on the use of one step transition probability matrices, which we refer to as the transition probability matrix method (TPM) [5, 1]. (See also $[6,7,9,10,11]$.)

The TPM has been applied to the problem of flow past a micro-plate. In lieu of experimental data, we have also applied the IP, DSMC and Navier-Stokes (NS) models to the same flows. The results are qualitatively similar and they provide some insight into the strengths and weaknesses of each of the models. We find broadly similar results for the drag coefficients and the IP and TPM results exhibit similar trends in velocity. As $K_{n}$ increases, the domain of influence of particles coming off the plate increases in a similar manner for both the IP and TPM. The NS slip model 


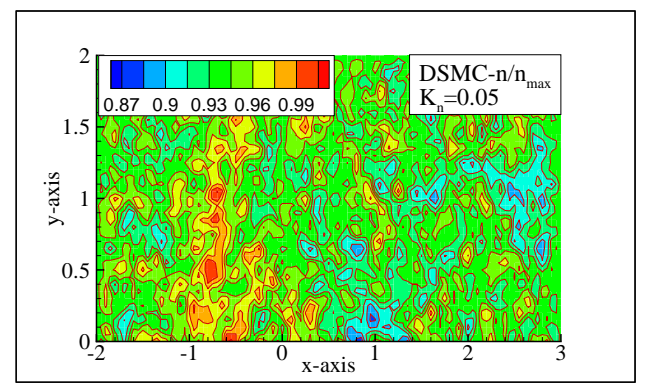

FIGURE 1. DSMC results for argon flowing past a flat plate. The inlet flow velocity is $\left(40 \frac{\mathrm{m}}{\mathrm{sec} .}, 0,0\right)$, the temperature is $288 K$ and the Knudsen number is 0.05 . The sampling size $\sim 3 \times 10^{5} \frac{\text { particles }}{\text { cell }}$ and the simulation domain consist of 4000 cells.

agrees with the IP and TPM for low $K_{n}$. As $K_{n}$ increases, the NS slip model fails to capture the essential physics . The density profiles for the NS slip model become more localized about the plate as $K_{n}$ increases and the velocity diverges from that predicted by both the TPM and IP models.

We begin with a brief introduction to the TPM followed by a discussion of the strengths and weaknesses of the TPM versus the IP and DSMC models. This is followed by a discussion of the results. Finally, we summarize the results and give some concluding remarks.

\section{TRANSITION PROBABILITY MATRIX (TPM) METHOD}

In this section we provide an overview of the TPM [1]. The objective of the TPM is to solve the steady state Boltzmann equation for LMFP environments,

$$
\vec{v} \cdot \nabla_{r} f(\vec{r}, \vec{v})+\frac{\vec{F}}{m} \cdot \nabla_{v} f(\vec{r}, \vec{v})=\left.\frac{\delta f}{\delta t}\right|_{\text {collision }}
$$

Instead of directly computing $f(\vec{r}, \vec{v})$, the TPM traces the collision rates, $R(\mathbf{c}, \mathbf{a}, E)$, of particles for each location, (position, direction, energy), of a $6 D$ phase space mesh [1]. The computation of $R(\mathbf{c}, \mathbf{a}, E)$ is performed through successive application of a one-step transition probability matrix,

$$
R(\mathbf{c}, \mathbf{a}, E)=\sum_{\mathbf{c}^{\prime}} \sum_{\mathbf{a}^{\prime}} \sum_{E^{\prime}} T\left(\mathbf{c}, \mathbf{a}, E: \mathbf{c}^{\prime}, \mathbf{a}^{\prime}, E^{\prime}\right) R\left(\mathbf{c}^{\prime}, \mathbf{a}^{\prime}, E^{\prime}\right)
$$

where $(\mathbf{c}, \mathbf{a}, E)$ are the coordinates of a phase space location. $R\left(\mathbf{c}^{\prime}, \mathbf{a}^{\prime}, E^{\prime}\right)$ is the number of particles that collided at coordinates $\left(\mathbf{c}^{\prime}, \mathbf{a}^{\prime}, E^{\prime}\right)$ at the previous iteration and $T\left(\mathbf{c}, \mathbf{a}, E: \mathbf{c}^{\prime}, \mathbf{a}^{\prime}, E^{\prime}\right)$ is the probability that a particle starting at coordinate $\left(\mathbf{c}^{\prime}, \mathbf{a}^{\prime}, E^{\prime}\right)$ will have its next collision at $(\mathbf{c}, \mathbf{a}, E)$. Given the other physical parameters such as the mean free path $(\lambda(\mathbf{c}, E))$, the particle velocity $(v(E))$, and so on, $R$ can be used to reconstruct any desired information about the flow. For example the density $n(\mathbf{c})$ is

$$
n(\mathbf{c})=\sum_{E} \frac{R(\mathbf{c}, E) \lambda(\mathbf{c}, E)}{|v(E)| \gamma(\mathbf{c})},
$$

where $n(\mathbf{c})$ is the density in cell $\mathbf{c}$ of the spatial mesh, $R(\mathbf{c}, E)=\sum_{\mathbf{a}} R(\mathbf{c}, \mathbf{a}, E)$, and $\gamma(\mathbf{c})$ is the volume of cell $\mathbf{c}$.

The transport and redistribution, in energy and angle, described by $T$ in equation 2 is broken up into two distinct operations, a ballistic move operation and a collisional redistribute operation, i.e., $T(R)=T_{c o l} \circ T_{b a l}(R) . T_{b a l}\left(\mathbf{c}, \mathbf{a}^{\prime}, E^{\prime}\right.$ : $\left.\mathbf{c}^{\prime}, \mathbf{a}^{\prime}, E^{\prime}\right)$ is the probability that a particle starting at a spatial location $\mathbf{c}^{\prime}$ moving along a direction $\mathbf{a}^{\prime}$ with energy $E^{\prime}$ will have its next collision in spatial cell $\mathbf{c} . T_{c o l}\left(\mathbf{c}, \mathbf{a}, E: \mathbf{c}, \mathbf{a}^{\prime}, E^{\prime}\right)$ is the probability that a particle that had a collision in cell $\mathbf{c}$ and was initially moving along the direction $\mathbf{a}^{\prime}$ at energy $E^{\prime}$ is redistributed with direction $\mathbf{a}$ and energy $E$. The equations for constructing $R(\mathbf{c}, \mathbf{a}, E)$, using $T_{b a l}$ and $T_{c o l}$, are,

$$
\begin{aligned}
R\left(\mathbf{c}, \mathbf{a}^{\prime}, E^{\prime}\right) & =\sum_{\mathbf{c}^{\prime}} R\left(\mathbf{c}^{\prime}, \mathbf{a}^{\prime}, E^{\prime}\right) T_{b a l}\left(\mathbf{c}, \mathbf{a}^{\prime}, E^{\prime}: \mathbf{c}^{\prime}, \mathbf{a}^{\prime}, E^{\prime}\right) \\
R(\mathbf{c}, \mathbf{a}, E) & =\sum_{\mathbf{a}^{\prime}} \sum_{E^{\prime}} R\left(\mathbf{c}, \mathbf{a}^{\prime}, E^{\prime}\right) T_{c o l}\left(\mathbf{c}, \mathbf{a}, E: \mathbf{c}, \mathbf{a}^{\prime}, E^{\prime}\right) .
\end{aligned}
$$


The ballistic operation, $T_{b a l}$, can be performed efficiently through the use of a 'propagating structure' and local phase space information about the flow at each cell $\mathbf{c}$. The propagating structure contains geometrical information about fractional overlaps and average lengths. A fractional overlap, $\gamma\left(\mathbf{c}, \mathbf{a}^{\prime}\right)$, is the fraction of particles that will pass though $\mathbf{c}$ having started in cell $\mathbf{c}^{\prime}$ with direction $\mathbf{a}^{\prime}$. The average length, $\left\langle L\left(\mathbf{c}, \mathbf{a}^{\prime}\right)\right\rangle$, is the average distance a particle will travel when passing through cell $\mathbf{c}$, so the number of particles distributed from location $\left(\mathbf{c}^{\prime}, \mathbf{a}^{\prime}, E^{\prime}\right)$ to location $\left(\mathbf{c}, \mathbf{a}^{\prime}, E^{\prime}\right)$ is given by

$$
N\left(\mathbf{c}, \mathbf{a}^{\prime}, E^{\prime}\right)=n\left(\mathbf{c}^{\prime}, \mathbf{a}^{\prime}, E^{\prime}\right) \gamma\left(\mathbf{c}, \mathbf{a}^{\prime}\right)\left(1-e^{\frac{\lambda\left(c, E^{\prime}\right)}{\left(L\left(\mathbf{c}, \mathbf{a}^{\prime}\right)\right\rangle}}\right)
$$

where $n\left(\mathbf{c}^{\prime}, \mathbf{a}^{\prime}, E^{\prime}\right)$ is the number of particles remaining at phase space location $\left(\mathbf{c}^{\prime}, \mathbf{a}^{\prime}, E^{\prime}\right)$ when the propagator reaches spatial location c. $R\left(\mathbf{c}, \mathbf{a}^{\prime}, E^{\prime}\right)$ is the sum of equation 6 over all $\mathbf{c}^{\prime}$.

$T_{c o l}$ redistributes particles in energy and direction while strictly enforcing energy and momentum conservation. The current version of $T_{c o l}$ employs one of two functions for redistribution in energy $(f(E))$, either a mono-energetic collision operator or a modified BGK collision operator [5]. Then redistribution in direction is performed through the use of a polynomial,

$$
f(\mathbf{a})=\left(1+\alpha v_{x}(\mathbf{a}) / v+\beta v_{y}(\mathbf{a}) / v+\gamma v_{z}(\mathbf{a}) / v\right),
$$

where $v$ is the speed of the outgoing particles, $v_{x}(\mathbf{a}), v_{y}(\mathbf{a})$ and $v_{z}(\mathbf{a})$ are the components of velocity of the particles moving in the direction a and $\alpha, \beta$, and $\gamma$ are normalization factors. The distribution $f(\mathbf{a})$ must total to one, i.e., $\int f(\mathbf{a}) d \mathbf{a}=1$. The integral provides the constraint needed to determine $\alpha, \beta$, and $\gamma$. Details regarding $f(E)$ can be found in reference [5] and details of $f(\mathbf{a})$ can be found in reference [7].

In the past, we have applied the TPM to $3 D$ arbitrary spatial meshes for $6 D$ phase space meshes [5]. However, in the current version, we have implemented the TPM for a uniform spatial mesh, for two reasons. It was discovered that in order to get uniform coverage of a spatial mesh for particles reflecting off walls, the earlier version of the TPM (in reference [5]) needed a 'volume reflecting' region behind the wall for tracking particle transport [1]. This volume reflecting region ensured that particles coming off the wall behaved as if they were coming from sources behind the wall. Without such a region the best that the method could achieve was a $1 \%$ variation in uniformity, which is on the order of the density variations for these high $K_{n}$ low $M$ flows we are interested in describing. With the volume reflecting region, uniformity variations were under $10^{-5}$. The version used here and in reference [1] does not require a volume reflecting region, however the results presented here make use of one. In addition, $T_{b a l}$ depends heavily on geometric information about the mesh, so a uniform mesh allowed for reduction in computational overhead by taking advantage of symmetry.

\section{A COMPARISON OF THE MODELS}

Generally, DSMC is one of the most successful particle methods for rarefied gas flows. It is several orders of magnitude less numerically expensive than methods for directly solving the Boltzmann equation, and several orders of magnitude more expensive than conventional CFD schemes. The DSMC method involves statistical scatter comparable to the mean thermal velocity of the molecules, i.e., the scatter per particle is $\sigma=\sqrt{2 R T}$ so that the scatter per cell is $\sigma^{\prime}=\frac{\sigma}{\sqrt{N}}$. In the previous two expressions $T$ is in Kelvin and $N$ is the number of samples per cell. Hence, it requires a huge number of samples for low speed gas flows, and becomes almost impossible for very low speed flows.

The information preservation method was proposed to solve the sampling difficulty in the DSMC method. In IP, particles simulated in the DSMC method additionally preserve macroscopic information about the flow field. This macroscopic information is updated during collisions between particles and collisions between particles and walls, and is modified to include the pressure effects exclude in the collisions. Namely, the macroscopic information preserved in the particles is updated according to the average behavior of the represented molecules during collisions while the pressure field effects are evaluated employing a continuum model to modify the macroscopic information contained in all particles in a given cell. The flow field is sampled from the macroscopic information preserved in the particles. This process dramatically reduces the statistical noise of the particle method for very low speed gas flows. It was shown [4] that the IP method, with models for updating the flow field, works very well for low speed gas flows ranging from the continuum regime to the free molecular regime. It was also mentioned that modified models were required for flows where the flow speed is not small. However, it is not very clear about the physics lost in the collision process for the preserved macroscopic information. Hence, it is suspected the current models are not physically correct for very high Knudsen number flows, but the results appear to support its validity for these low speed flows. 
The Navier-Stokes equations are solved using a finite volume formulation [12]. The fluxes are evaluated with a second-order accurate modified Steger- Warming flux-vector splitting approach, and an implicit Gauss-Seidel linerelaxation method is used for the time integration. Compared with particle methods, the NS solver is relatively fast. However, the Navier- Stokes equations are only accurate for near equilibrium flows. To enhance the validity of the equations, a Maxwellian type slip wall model is used. Then, the Navier-Stokes solver may be applied to flows with Knudsen number up to 0.1 .

The TPM was proposed as an alternative to statistical particle transport models. The drawback to this version of the TPM is that the BGK model becomes of questionable validity for $K_{n}>1.0$. However, given the differential crosssection for a desired species, the TPM could easily be altered to handle collisions exactly for high $K_{n}$ flows. Another alternative to the BGK model is to use a DSMC model to determine the statistics for collisions [7]. However, in a flow where there were wide variations in density, pre-computing the collision rates with a DSMC model would not be possible.

All four models have limitations. For DSMC, low speed flows present difficulties because of the enormous number of samples needed to reduce statistical noise. DSMC could be effective for these low speed flows, if enough computing power were available. However this is unlikely to happen soon.

For the IP method, it is not clear that the continuum models currently used are well suited for high $K_{n}$. However, numerical results of the IP model for simple high $K_{n}$ test flows are in good agreement with DSMC results [4]. Some thought needs to be given to understanding what is the essential physics that is lost in the IP during the averaging process. This may provide the key to being able to propose models for building back the 'lost' physics.

The NS slip model does not capture the essential physics of high $K_{n}$ environments. Higher order corrections to the NS model, such as the Burnett equations, could be used but this does not address the fundamental issue that most high $K_{n}$ flows are not well described by a single macroscopic flow velocity and temperature.

The TPM may be the approach that is easiest to modify in order to model high $K_{n}$ flows, because it is well understood what additional physics needs to be included to provide a more complete collision model.

\section{RESULTS}

In this section, the results for the TPM, IP and NS simulations, for flow past a flat plate, are presented and discussed. All of the flows presented are for argon. The far field boundary conditions (an inlet velocity set to $\left(v_{x}=40 \frac{m}{s e c}, v_{y}=\right.$ $\left.0 \frac{m}{s e c .}, v_{z}=0 \frac{m}{s e c .}\right)$ ) are used for all the runs presented. Although these boundary conditions are not physical for the flow domains investigated, these conditions were used because they make direct comparison of the models easier. Since the speed of sound in argon is around $316 \frac{\mathrm{m}}{\mathrm{sec}}, M \sim 0.12$. The flows presented for all three cases are for Knudsen numbers $\left(\frac{\lambda}{L}\right)$ of $0.05,0.2$ and 1.2. In the figures the first columns are the normalized density contours, the second columns are the normalized $\mathrm{x}$-velocity contours and the the third columns are the normalized y-velocity contours for the TPM, IP and NS models. We also compare the drag coefficient, $C_{d}$, for $K_{n}$ of $0.05,0.2,0.8$, and 1.2 as predicted by these models. In addition, we present TPM results for $K_{n}=10$.

Figure 2 is for $K_{n}=0.05$. The upstream region is $1 \frac{1}{2}$ times the length of the plate, the downstream region is $2 \frac{1}{2}$ times the length of the plate and the height of the simulation domain is 2 times the length of the plate. The simulations were run on two different meshes. The first mesh used was the same for all three simulations to make comparisons easier. This first mesh had a uniform spacing of a mean free path, i.e., $\Delta M=\langle\lambda(\mathbf{c}, E)\rangle$. The second mesh used by the IP and NS models was non-uniform with many cells packed around the plate, while the second mesh for TPM was a uniform mesh with double the resolution of the first uniform mesh. Except at the upstream boundary, where the artificial fixing of the density causes unphysical behavior in the IP and NS results, the density contours exhibit similar trends in all cases. The maximum and minimum densities determined by the TPM, IP and NS for the uniform mesh are $(1.012,0.988),(1.035,0.975)$, and $(1.029,0.977)$. When the TPM was run on the second uniform mesh, the maximum and minimum values are $(1.019,0.981)$. For a non-uniform mesh, the IP and NS find a maximum and minimum density of $(1.020,0.980)$ and $(1.034,0.973)$. The values for maximum and minimum density for the three models with better resolution are comparable although the NS gives a somewhat larger variation. The normalized velocities for all three models also exhibit similar behavior. For the cases shown in figure 2, the minimum and maximum $\mathrm{x}$-velocities are $(0.11,1.20)_{T P M},(0.22,1.18)_{I P}$, and $(0.22,1.16)_{N S}$. The y-velocity ranges are $(-0.16,0.17)_{T P M},(-0.12,0.17)_{I P}$, and $(-0.17,0.17)_{N S}$. Only the x-velocities exhibit much change when the mesh is altered. The $\mathrm{x}$-velocity range for the TPM, on a mesh with double the resolution, is $(0.13,1.15)$. For the IP and NS models the ranges are $(0.13,1.07)$ and $(0.16,1.14)$. Table 1 shows the calculated drag coefficient for the DSMC, IP, NS and TPM models. The DSMC results 


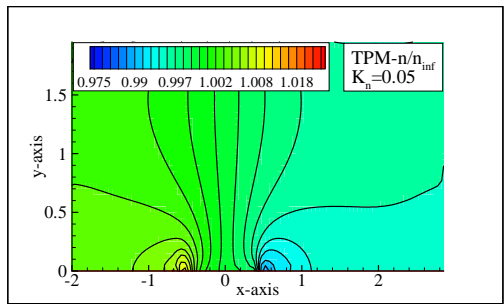

a)

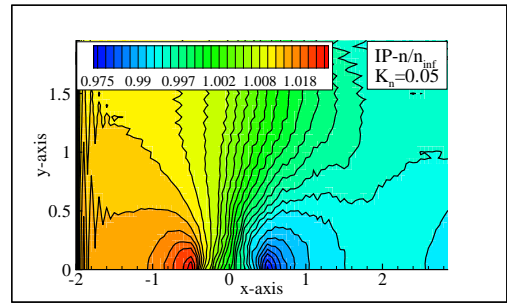

b)

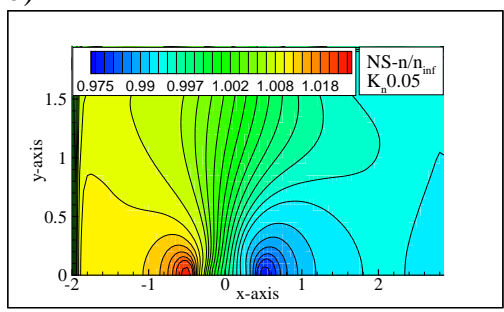

c)

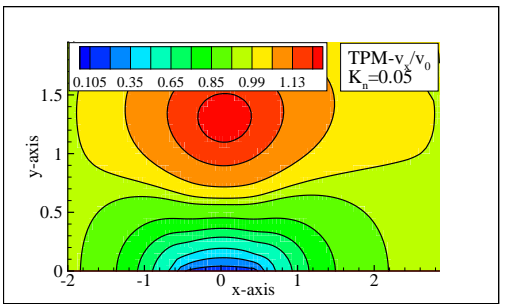

d)

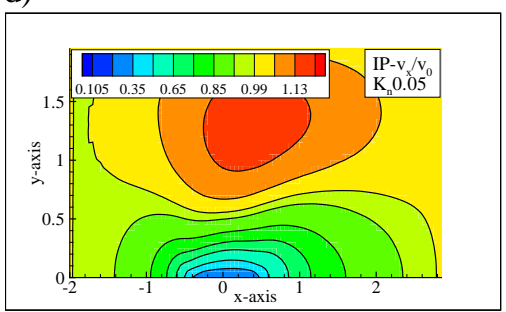

e)

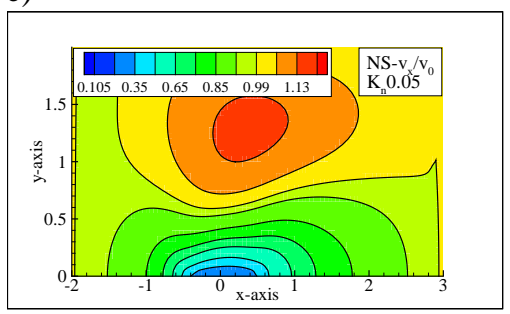

f)

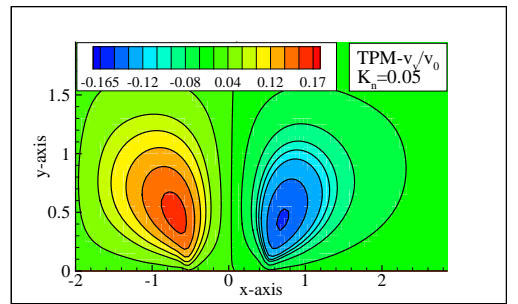

g)

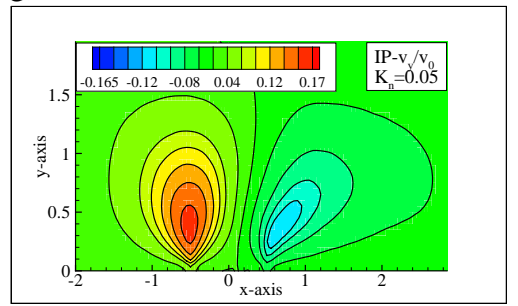

h)

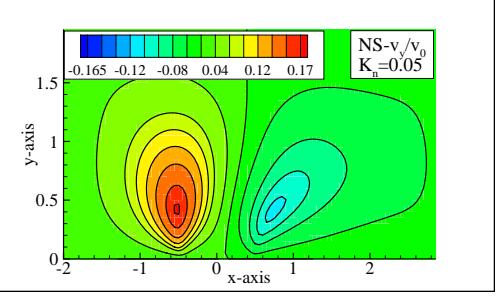

i)

FIGURE 2. Results for flow past a flat plate for the TPM, IP and NS models generated with a uniform grid. The flow is argon with an inlet velocity of $(40 \mathrm{~m} / \mathrm{sec}, 0,0)$ and $K_{n}=0.05$. The first column is normalized density. The second column is the normalized $\mathrm{x}$ velocity and the third column is the normalized y velocity.

TABLE 1. Drag coefficient for flow past a flat plate for the DSMC , IP, NS and TPM models. In all cases $K_{n}=0.05$.

\begin{tabular}{c|ccccc} 
Kn & grid & DSMC & IP & NS & TPM \\
\hline 0.05 & small,uniform1 & 1.84 & 1.89 & 1.70 & 2.30 \\
0.05 & small,uniform2 & - & - & - & 1.65 \\
0.05 & small,non-uniform & 1.64 & 1.62 & 1.80 & - \\
0.05 & large,non-uniform & 1.52 & 1.45 & 1.52 & -
\end{tabular}

are given here for comparison purposes, although the statistical scatter is not small enough. The column designated grid specifies the type of mesh that the results were generated on. 'small, uniform1' designates the drag coefficient for the plots shown in figure 2. 'small, uniform2' is the same domain size as mentioned above but with double the resolution of the mesh. 'small, non-uniform' is the same domain size as mentioned above but the results are for a non-uniform mesh. 'large, non-uniform' is about three times the domain size as mentioned above and makes use of a non-uniform mesh. Our first observation is that the drag coefficient is dependent on the resolution of the mesh. The first row of table 1 shows that, for identical boundary conditions and mesh spacing, the TPM predicts the highest drag coefficient $\left(C_{d}=2.30\right)$ while the NS gives the lowest drag coefficient $\left(C_{d}=1.70\right)$ for these conditions. For a higher resolution mesh near the plate and a smaller domain, the DSMC, IP and TPM models are in good agreement, whereas the NS model predicts a value higher than what the model computed for the uniform mesh (as can be seen in rows 2 and 3 of table 1). However, the NS model agrees well with the results for the DSMC and IP methods for the larger domain, see row 4 of table 1.

Figure 3 shows the density, $\mathrm{x}$-velocity and y-velocity contours for $K_{n}=0.2$. The results shown in figure 3 are for two different meshes. The TPM was run on a uniform mesh with a mesh spacing of $\Delta M=\frac{\langle\lambda(\mathbf{c}, E)\rangle}{2}$. The IP and NS models are for a non-uniform mesh that packs cells around the plate. The density contours exhibit similar behavior. The 


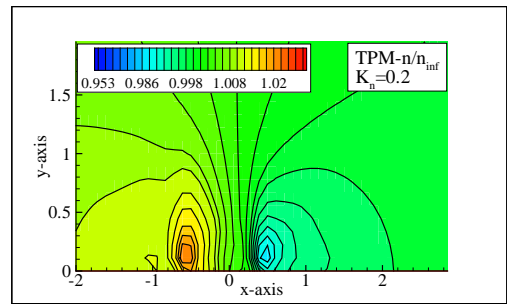

a)

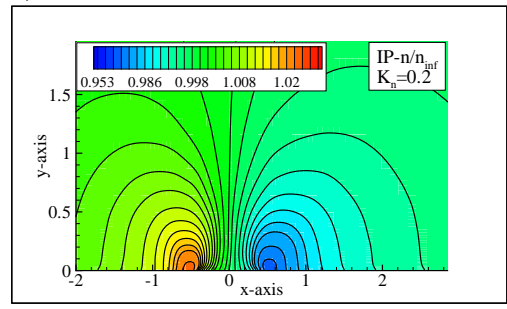

b)

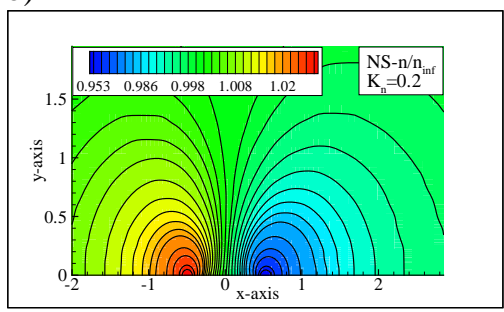

c)

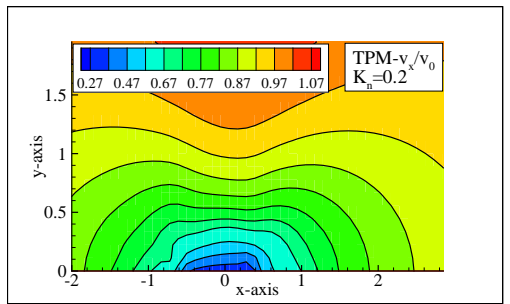

d)

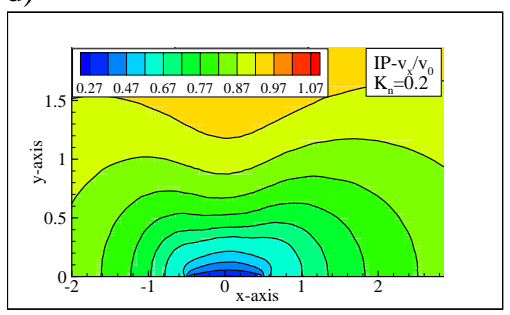

e)

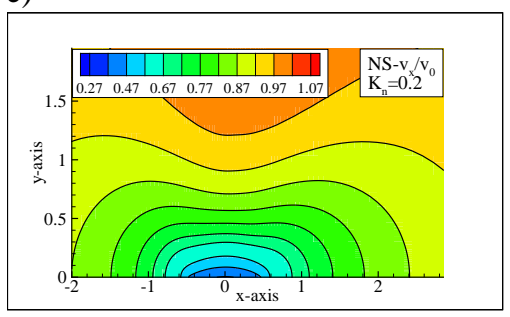

f)

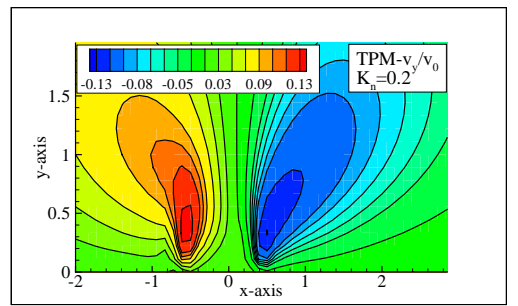

g)

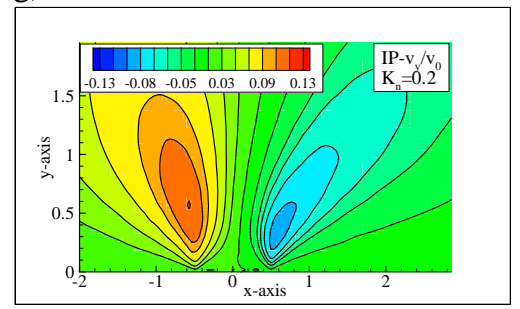

h)

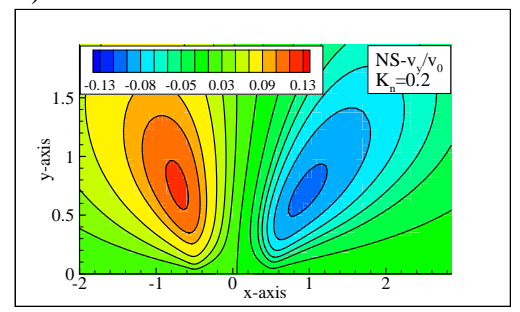

i)

FIGURE 3. Results for flow past a flat plate for the TPM, IP and NS models. The flow is argon with an inlet velocity of $(40 \mathrm{~m} / \mathrm{sec}, 0,0)$ and $K_{n}=0.2$. The first column is normalized density. The second column is the normalized x velocity and the third column is the normalized y velocity.

maximum and minimum densities determined by the TPM, IP and NS models are $(1.022,0.985),(1.028,0.972)$, and $(1.062,0.942)$. The peak density computed by the TPM is slightly off the surface of the plate, where as the IP model predicates that the maximum and minimum density should be at the surface of the plate. The normalized velocities for all three models also exhibit similar behavior. For the cases shown in figure 2, the minimum and maximum $\mathrm{x}$-velocities are $(0.28,1.07)_{T P M},(0.26,1.02)_{I P}$, and $(0.36,1.03)_{N S}$. The y-velocity ranges are $(-0.13,0.14)_{T P M},(-0.09,0.11)_{I P}$, and $(-0.11,0.11)_{N S}$.

Figure 4 is for $K_{n}=1.2$. The upstream region is 5 times the length of the plate, the downstream region is 7 times the length of the plate and the height of the simulation domain is 4 times the length of the plate. The TPM results shown in figure 4 are for a uniform mesh while the results for the IP and NS models shown in figure 4 are for a non-uniform mesh. The density contours for $K_{n}$ of 1.2 are not as similar as for the lower $K_{n}$, but the three models have their minimum and maximum densities in about the same location. The maximum and minimum densities determined by the TPM, IP and NS for the uniform mesh are $(1.022,0.978),(1.038,0.966)$, and $(1.048,0.956)$. Both the $\mathrm{x}$ and $\mathrm{y}$ velocity contours for the TPM and IP are very similar, while the NS results are not. For the results shown in figure 4, the minimum and maximum $\mathrm{x}$-velocities are $(0.5,1.02)_{T P M},(0.46,1.0)_{I P}$, and $(0.70,1.0)_{N S}$. The y-velocity ranges are $(-0.12,0.12)_{T P M},(-0.092,0.092)_{I P}$, and $(-0.018,0.018)_{N S}$. It is not surprising that the NS slip model results are not very similar to the kinetic models for such a high $K_{n}$, since this is well outside the range of $K_{n}$ where the NS model is applicable. The IP results for the same uniform mesh spacing, used for the TPM, have the same general shape but the minimum and maximum values differ from the IP or TPM results. The density range for the uniform mesh IP is $(1.01,0.99)$ and $\mathrm{x}$ and $\mathrm{y}$-velocity ranges are $(0.57,1.0)_{x}$ and $(-0.016,0.016)_{y}$.

Figure 5 is for $K_{n}=10$. The results were generated with the TPM. The upstream region is 5 times the length of the plate, the downstream region is 7 times the length of the plate and the height of the simulation domain is 4 times the length of the plate. The maximum and minimum densities are $(1.03,0.96)$. The minimum and maximum velocities are $(0.55,1.01)_{x}$ and $(-0.20,0.21)_{y}$. All of the contours are more striated than in the previous cases. It is not clear at 


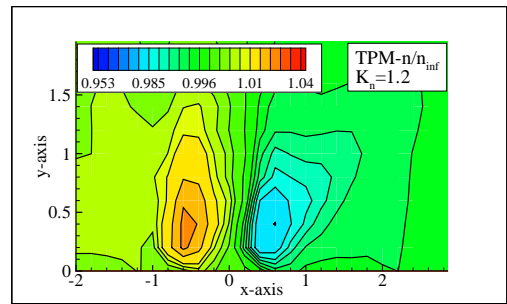

a)

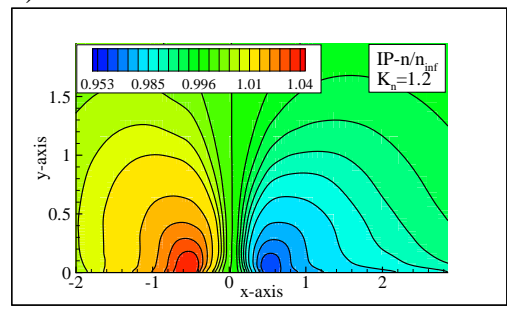

b)

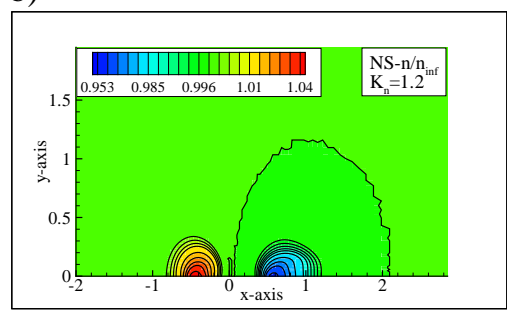

c)

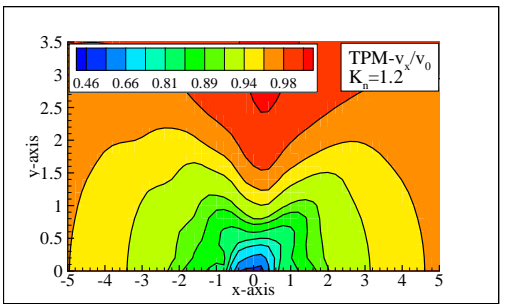

d)

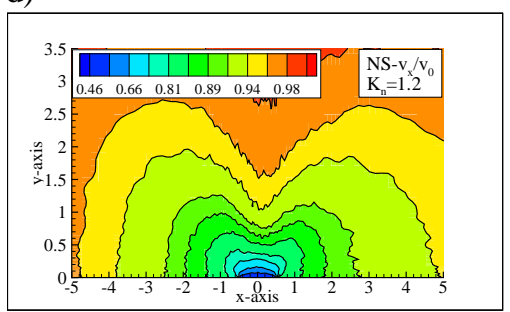

e)

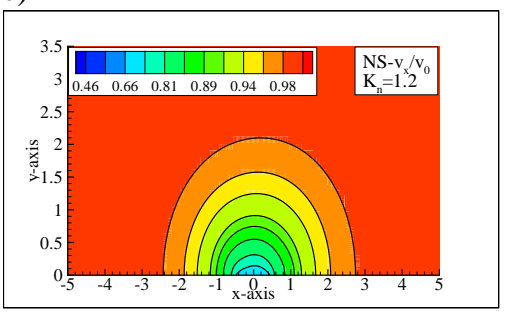

f)

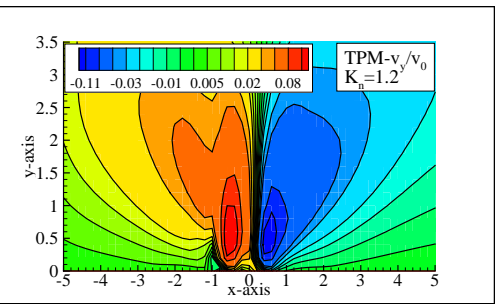

g)

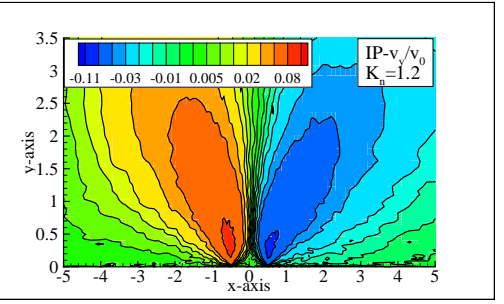

h)

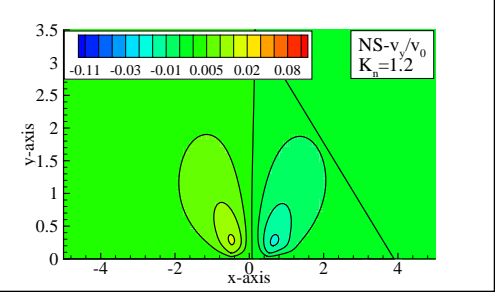

i)

FIGURE 4. Results for flow past a flat plate for the TPM, IP and NS models. The flow is argon with an inlet velocity of $(40 \mathrm{~m} / \mathrm{sec}, 0,0)$ and $K_{n}=1.2$. The first column is normalized density. The second column is the normalized x velocity and the third column is the normalized y velocity.

a)

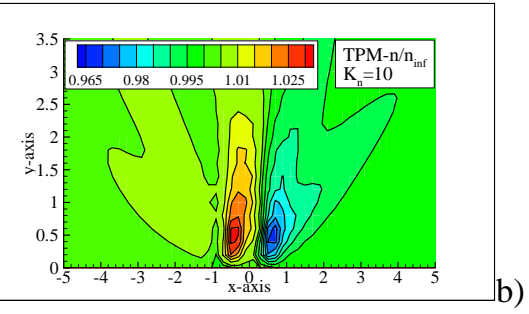

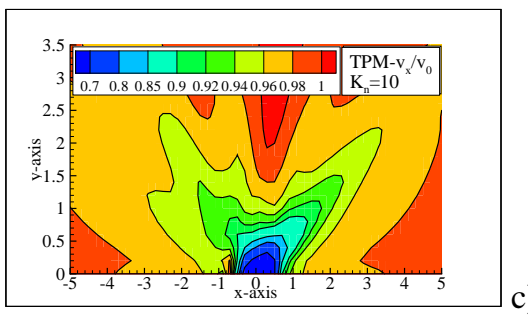

c)

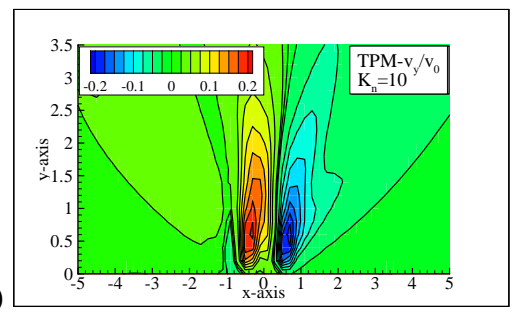

FIGURE 5. Results for flow past a flat plate for the TPM model. The flow is argon with an inlet velocity of $(40 \mathrm{~m} / \mathrm{sec}, 0,0)$ and $K_{n}=10.0$. Figure a) is the normalized density. Figure b) is the normalized x velocity and figure c) is the normalized y velocity.

this point if this is a resolution effect; doubling the angular resolution had little effect on the results.

Table 2 shows the drag coefficients calculated for various $K_{n}$ on several different meshes. The uniform grids were the same to make comparison between TPM and IP easy. The designation $S$, in the table, specifies an upstream length of $1 \frac{1}{2}$ plates, a downstream region of $2 \frac{1}{2}$ and a simulation height of 2 plate lengths. The designation $S 2$ is the same as $S$ but with double the resolution on the mesh. The designation $L$ specifies an upstream length of 5 plates, a downstream region of 7 and a simulation height of 4 plate lengths. The table also includes the drag coefficient for non-uniform grids. The free molecular flow drag coefficient for argon under these flow condition is $C_{d}=4.88$. The drag coefficient determined by the IP and DSMC simulations for a uniform mesh is an overestimate for 0.8 and 1.2 . This may be a result of the unphysical boundary conditions which could cause some regions of the domain to have a larger momentum than they should in order to balance the momentum loss to the plate, which would result in the particles hitting the plate having a larger momentum than expected. Comparing the TPM results to the non-uniform IP and DSMC results we see that the TPM agrees with the IP and DSMC for a $K_{n}=0.2$ and predicts a smaller drag coefficient for higher $K_{n}$. 
TABLE 2. Drag coefficient for flow past a flat plate for the DSMC, IP, and TPM models.

\begin{tabular}{c|cccc|ccc}
$K_{n}$ & grid-(uniform) & TPM & IP & DSMC & grid-(non-uniform) & IP & DSMC \\
\hline 0.05 & S & 2.30 & 1.89 & 1.84 & S & 1.62 & 1.64 \\
0.05 & S2 & 1.65 & - & - & L & 1.45 & 1.52 \\
0.2 & L & 3.08 & 3.22 & 2.99 & L & 3.00 & 3.04 \\
0.8 & $\mathrm{~L}$ & 3.56 & 5.05 & 4.55 & $\mathrm{~L}$ & 4.62 & 4.25 \\
1.2 & $\mathrm{~L}$ & 3.90 & 5.42 & 5.05 & $\mathrm{~L}$ & 4.81 & 4.76 \\
10.0 & $\mathrm{~L}$ & 4.39 & - & - & - & - & -
\end{tabular}

This may be due to mesh size effects. It is also worth noting that the TPM predicts a $C_{d}$ less than the free molecular flow value for $K_{n}=10$, which is probably because $f(\mathbf{a})$ (equation 7) gives the correct drift velocity but underestimates the transverse momentum-flux to the surface.

\section{CONCLUDING REMARKS}

The problem of accurately simulating low Mach number high Knudsen number flows is an interesting challenge. We have given an overview of the approach we are developing (the TPM) for this problem and have presented results for the TPM model for flow past a micro-plate for various $K_{n}$. In addition, we have presented results for two other models, the IP and NS models, for the same range of $K_{n}$. The results for all three models exhibit similar trends, i.e. the normalized minimum and maximum densities increase as $K_{n}$ increases and the difference in the minimum and maximum velocities decreases as $K_{n}$ increases.

Future work will involve extending the TPM to diatomic gas, flow past an object for any angle of attack, exploring other collision models, and investigating how to implement irregular meshes in an efficient manner. In addition, comparisons of all methods will be made with new experimental data being generated for micro-scale airfoils being conducted in a micro-scale wind-tunnel [13].

\section{ACKNOWLEDGMENTS}

We would like to thank the Air Force Office of Scientific Research for supporting the research under grant number F49620-98-1-0433.

\section{REFERENCES}

1. Christlieb, A., and Hitchon, W., Conference paper presented at the $23^{\text {rd }}$ Symposium on Rarefied Gas Dynamics (Whistler, BC, Canada, July 20-25, 2002).

2. Sun, Q., Boyd, I., and Candler, G., Journal of Thermophysics and Heat Transfer, 16(2), 171 (2002).

3. Boyd, I., and Sun, Q., 39th AIAA Aerospace Science Meeting, Reno Nevada (January, 2001).

4. Sun, Q., and Boyd, I., Journal of Computational Physics, 179, 400 (2002).

5. Christlieb, A., and Hitchon, W., Physical Review E, 65, Article 056708 (2002).

6. Harvey, R., Hitchon, W., and Parker, G., Journal of Applied Physics, 75, 1940 (1994).

7. Parker, G., Hitchon, W., and Keiter, E., Physical Review E, 54, 938 (1996).

8. Fan, J., and Shen, C., 167, 393 (2001).

9. Askew, J., Journal of British Nuclear Energy Society, 5, 564 (1966).

10. Vujic, J., Proceedings of the International Conference on Mathematical Methods and Supercomputing in Nuclear Applications, Karlsruhe. (1993).

11. Cale, T., Raupp, G., and Gandy, T., Journal of Applied Physics, 68, 3645 (1990).

12. MacCormack, R., and Candler, G., 17(1), 135 (1989).

13. Martin, M., Boyd, I., and Bernal, L., Proceedings of 2002 ASME Fluids Engineering Division Summer Meeting (New York, NY. July 2002). 